\title{
Le fait religieux aux Antilles et la règle fondamentale de la catholicité
}

The religious phenomenon in the French West Indies and the fundamental rule

of Catholicity

Dominique Aimé Mignot

\section{(2) OpenEdition}

\section{Journals}

Édition électronique

URL : http://journals.openedition.org/plc/200

DOI : $10.4000 /$ plc. 200

ISSN : 2117-5209

Éditeur

L'Harmattan

Édition imprimée

Date de publication : 15 décembre 2007

Pagination : 249-268

ISBN : 2-296-01986-2

ISSN : 1279-8657

\section{Référence électronique}

Dominique Aimé Mignot, « Le fait religieux aux Antilles et la règle fondamentale de la catholicité »,

Pouvoirs dans la Caraibe [En ligne], 15 | 2007, mis en ligne le 06 mai 2011, consulté le 19 avril 2019

URL : http://journals.openedition.org/plc/200 ; DOI : 10.4000/plc.200 


\title{
LE FAIT RELIGIEUX AUX ANTILLES ET LA REGLE FONDAMENTALE DE LA CATHOLICITE ${ }^{1}$
}

\author{
Dominique Aimé MIGNOT \\ Docteur de III cycle \\ Docteur d'Etat (droit romain) \\ Membre de l'AFHIP (Aix-en-Provence) et \\ du GIREA (Besançon) \\ Université des Antilles et de la Guyane
}

\begin{abstract}
Nous reprenons ici les principaux développements d'une conférence donnée au C.A.G.I. le 12 avril 2002.

Il est vrai que l'histoire des Antilles françaises, pour complexe qu'elle soit compte tenu de la situation géographique de l'archipel et du contexte de guerres sur fond économique et stratégique qui opposèrent les puissances anglaise et française, ne se réduit pas seulement à une simple succession d'évènements politiques et militaires importants mais également postule une lente pénétration religieuse et spirituelle, fruit de l'activité des premiers missionnaires.
\end{abstract}

Le fait est là. Le développement des Antilles traduit l'évolution de la civilisation occidentale sous des dehors et aspects brutaux. Nous sommes en présence d'une histoire coloniale. Et peu importe la nature juridique de cette colonisation en ses débuts. Une société mère, le royaume de France, entreprend au début du XVII ${ }^{\mathrm{e}}$ siècle une oeuvre dite de civilisation en Amérique centrale, sans disputer aux hispano-américains - au Sud - ni aux Anglais et Hollandais - au Nord - les places dans lesquelles ils étaient installés depuis plus de cent ans.

Le trop plein des forces vives du royaume, les populations remuantes et indésirables sont appelées à visiter et à s'installer dans des contrées lointaines et à reproduire, nolens volens, le schéma politique et institutionnel de la France. On note comme à Athènes (les clérouques) ou à Rome (les cités de droit latin) une expansion de type métastasique. Les cellules filles comprennent dans leurs gènes les caractères dominants de leurs ancêtres. On créera donc des comptoirs, puis des établissements ; enfin, dans le second versant du XVII ${ }^{\mathrm{e}}$ siècle les possessions françaises

\footnotetext{
${ }^{1}$ Nous reprenons ici les principaux développements d'une conférence donnée au Centre d'Analyse Géopolitique Internationale (UAG) le 12 avril 2002. Une version française de cette étude a été publiée à l'Academia Real de la Historia, Madrid, sous le titre : El hecho religioso en las antillas y la norma fundamental de catolicidad, tomo CCIL, cuaderno II, 217 - 233.
} 
intégreront le domaine royal. On quitte peu à peu la conception féodoseigneuriale des colonies à charte pour s'orienter vers une conception plus étatique. C'est qu'à partir de septembre 1661 la France est gouvernée par un Prince dont l'absolutisme princier (M. Prelot) s'exportera également vers les colonies d'Amérique (Nouvelle France, Petites et Grandes Antilles...).

Or, parmi ces caractères politiques dominants figurent tout naturellement les éléments économique et religieux, à savoir les fondements de cette société nouvelle. Autrement dit, quel fut, en conséquence, le statut des clercs aux Antilles ? Un auteur ${ }^{2}$ contemporain a rappelé dans sa belle thèse de doctorat que l'évangélisation des Caraïbes avait commencé par le martyre de la première mission chrétienne qui tenta de convertir ces derniers. Autre point important : quelles furent les rôles et compétences des religieux dans une société coloniale bigarrée inégalitaire et qui subit très tôt une véritable crise de l'esclavagisme au XVIII ${ }^{\mathrm{e}}$ siècle ?

Il importe d'examiner brièvement ici les deux fondements de l'ordre public colonial à savoir l'utilité économique et l'action missionnaire chrétienne fondée sur le principe fondamental de catholicité du royaume. C'est cet ordre public qui, à son tour, conditionne le statut des religieux.

Louis XIII et son Principal Ministre Richelieu n'envisagent une politique de développement ultra-marin économique et commercial que si cette dernière s'accompagne d'une politique d'expansionnisme religieux ${ }^{3}$. En 1626, Richelieu fonde la Compagnie des Indes par lectres officielles ou patentes : l'entreprise a pour but fondamental de "planter la foy chrestienne a la gloire de Dieu et l'honneur du roy [à condition de] de mener des prebstres et de cultiver et travailler a toutes sortes de mines et de metaulx 4 .». Il n'est pas encore question d'esclavage ! Faut-il voir là un

\footnotetext{
${ }^{2}$ L. R. Abeneon, La Guadeloupe de 1679 à 1759, th. Lettres, Paris, 1987, t. I, 50 ss.

3 V. de P. Chevillard, Les desseins de Son Eminence de Richelieu pour l'Amérique, reproduction de l'éd. de 1659, Soc. Hist. de la Guadeloupe, 1973.

${ }^{4}$ V. Extraicts du contrat de la Cie avec les SSrs de l'Olive et du Plessis, 12-14 févr. 1635 in Du Tertre, Histoire generale des Antes-isles habitees par les Franscois, Paris, 1667, (2 éd.) t. I, 88, cf. art. 1er Edit établissant la Cie des Indes Occidentales Du même : t. III, 76 : Elle sera obligée de faire passer aux paiis ci-dessus concédés, le nombre d'ecclesiastiques necessoire pour y prescher les saincts evangiles et instruire ces peuples en la croyance de la religion catholicque apostolicque et romaine, comme aussy de bastir des esglises et d'y establir des cures et prebstres ( dont elle aura la nomination) pour fayre service divin aulx jours et heures ordinaires et administrer les sacremens aulx abitants. L'art. 4 du contrat dispose que tous les colons doivent estre catholicques et franscois...
} 
caprice d'un monarque très puissant ? Ou encore comme d'aucuns l'ont soutenu, d'une vélléité de moralisation de la future entreprise coloniale française ? Certes on peut répondre hâtivement par la positive. C'est ce que firent de nombreux historiens qui considèrent que la motivation religieuse entendait donner un sens non seulement à la colonisation française mais surtout à l'acceptation de la servitude en Amérique, notamment lors de la publication du Code Noir Louis (édit de mars 1685) ${ }^{5}$.

Auguste Cochin a pu écrire que, « Sur la demande du cardinal de Richelieu, le pape Urbain VIII leva ces censures, et le 12 juillet 1635, [il] donna pouvoir à quatre religieux dominicains, sous la protection du roi de France, pouvoir qui lui fut confirmé plusieurs fois au même ordre, puis partagé avec plusieurs autres ». L'auteur rappelle que le P. Du Tertre fit partie du deuxième envoi de missionnaires. Ce dernier, chroniqueur de renom, précise que les PP. Pélican, Griffon, Nicolas ainsi que le P. Raymond, passèrent leur temps à se dévouer pour protéger et évangéliser les indiens caraïbes ${ }^{6}$. A. Cochin précise encore que, selon la chronique du même auteur, six religieux en 1603 et six en 1604 avaient trouvé la mort dans les Antilles et que «celles-ci eurent des martyrs, c'est-à-dire des témoins de la foi avant d'avoir des colons ${ }^{7} \gg$.

A l'analyse, les choses sont davantage complexes. Il s'agit pour le roi de France de rattraper un retard considérable dans l'œuvre colonisatrice $\mathrm{du}$ Nouveau Monde. Plus encore, il importe de n'accepter une telle entreprise que si elle est conforme aux Loix fondamentales du royaulme, c'est-à-dire à la norme même qui fonde par le sacre le pouvoir politique du souverain : la catholicité.

Or, le principe de catholicité suppose que le roi ne soumette pas son peuple à une religion particulière, nouvelle, qui ne soit pas dans le droit fil de la succession apostolique. Il ne peut pas y avoir religion du Prince mais seulement un Prince soumis à la religion de tous et de toujours et qui appartienne à la traditionnelle cattolica ${ }^{8}$. Dans cette optique on s'aperçoit de

\footnotetext{
${ }^{5}$ Une des meilleures éditions du Code est celle parue chez Prault, imprimeur de Mgr le Chancelier (Paris, 1742) contenant le texte stricto sensu ainsi qu'un Recueil des reglemens rendus jusqu'a present...

${ }^{6}$ Du Tertre, ouvr cit., 29.

${ }^{7}$ A. Cochin, L'abolition de l'esclavage, Paris, Désormeaux - L'Harmattan, 1979, $246-247$.

${ }^{8}$ Il n'y a pas de place en France pour le nouveau dogme politique né du protestantisme européen :
} 
la modestie du principe et des limites que les rois, et notamment Henri IV, se sont vu imposer. Ils ne peuvent agir comme des tyrans car eux-mêmes se soumettent non seulement aux dogmes de l'Eglise définis par les Pères au concile de Nicée mais surtout, ils ont les obligations et les devoirs de fils de l'Eglise. Hérétique! Schismatique ! Son pouvoir sur ses sujets ne sera alors plus légitime ${ }^{9}$. Assurément cette loi de catholicité constitue un frein à la monarchie absolue. Louis XIII ne pourra donc entrevoir de colonisation que si elle s'accompagne - ou plutôt complète une action missionnaire ${ }^{10}$. Tel est le résultat de la politique du Tiers-Etat lors des estats generaulx tenus en 1614-1615 après que deux rois de France eurent été assassinés en vingt et un ans, et surtout depuis le meurtre d'Henri IV par Ravaillac ${ }^{11}$. Le clergé fut surpris de se trouver devant un véritable projet de loi fondamentale écrit et rédigé en fait par les grandes robes parisiennes dont le grand-père maternel de Bossuet : le pouvoir royal serait confié au Prince directement par Dieu et non plus avec la réserve thomiste par le peuple: omnis potestas a Deo per populum $^{12}$. Louis XIII, d'ailleurs gêné, et par humilité, retira lui-même l'article du tiers défendu par l'auvergnat J. Savaron qui faisait de lui un rex diuini iuris, jugeant que le tiers-état n'avait point à juger de la foi du prince ni le pouvoir de créer un nouveau dogme. Cependant, en fait, c'est en vertu de l'édit d'avril 1615 que la règle de catholicité, de véritable coutume constitutionnelle posée par les parlementaires, devient la norme fondamentale du royaume, fondant la légitimité de l'action du Prince dans le

Cujus regio, hujus religio. De ce fait la loi fondamentale de catholicité interdit d'asseoir l'influence d'un Prince sur une nouvelle religion. La catholicité peut se définir comme universalité (Optat de Milev[e]) et Victor de Lerins lui donnent pour signe : quod ubique, quod semper, quod ad omnibus creditum est (Commonitorium.... II, 3).

${ }^{9}$ Cf. les célèbres édits dit d'Union sous Henri III et l'arrêt de règlement du président Lemaistre du 28 juin 1593 disposant : "La Cour, toutes chambres assemblées, n'ayant, comme elle n'a jamais eu, d'aultres intention que de maintenir la religion catholicque, apostolicque et romaine en l'Estat et couronne de France, soubz la protection d'un roy trèschrestien catholicque et fransçois. »

${ }^{10}$ Cf. Edit 23 avr. 1615, cf. C. N., art. I : «Voulons et entendons que l'edit du feu roy de glorieuse mesmoire nostre tres - honore seigneur et pere... soit execute dans noz isles... »

${ }^{11}$ En ce sens, R. Mousnier, Les institutions de la France...t. I, Paris, PUF, 508.

12 Ainsi, dans le projet de 1614-1615, MM. du parlement de Paris rejettent l'ancienne tradition, aussi bien la théorie de Bellarmin que celle de l'Université de Paris : "Il n'y a de puissance quelle qu'elle soict, spirituelle ou temporelle, quy ait aulcun droict sur le royaume pour priver les personnes sacreez de noz roys, ny dispenser ou absouldre leurs subjects de la fidelite et obeissance qu'ilz lui doivent pour quelque cause ou pretexte que ce soit ». - A. -G. Martimort, Le gallicanisme, Paris, PUF, 1973, 62 ss. 
royaume et ses dépendances. Dès lors, les Lettres de commission des différents généraux et gouverneurs feront quasiment toujours référence et de façon constante à la mission évangélisatrice : le duc d'Amville, ayant charge de vice-roi en juillet 1655 "doit commander en tous les paiis d'Amérique... establyr nostre auctorite... fayre obéir tous les peuples des dites terres... par toutes les voies les plus doulces qui se pourroient a la connoissance de Dieu a la lumière de la foy et a la religion Catholicque, Apostolicque et Romaine, y establir l'exercice a l'exclusion de tout aultre ${ }^{13}$ »; il faudra donc emporter dans les bateaux des vivres, des hommes, des canons et des munitions, certes, mais également des hommes d'Eglise pour convertir, baptiser, instruire, contrôler aussi l'action des colons, et ainsi exercer une véritable cura morum sur tous les êtres vivants, libres ou non, civils ou militaires, installés si loin des côtes françaises...

C'est sans doute en raison de ce principe de catholicité que déjà, dès les années 1660-1670 on songe à modifier l'organisation administrative des Ante-isles d'Amérique. Dès cette époque un fait social nouveau, d'une ampleur inconnue des régnicoles de la métropole, prend corps : l'institution des engagés tournant court il faut importer dans ces contrées torrides des tropiques des hommes venus d'Afrique pour y accomplir le travail de la terre. De façon anarchique, l'institution de l'esclavage s'y répand de 1650 à $1685^{14}$. Et tout cela sans contrôle bien apparent hormis quelques règlements pris par les Conseils souverains de Martinique et supérieur de la Guadeloupe...

\footnotetext{
${ }^{13}$ Provisions données à Laferre, au mois de juillet, l'an de grasce 1655 et de nostre regne le treizieme, signé Louis et plus bas, Par le roy, De Loménie et scellé du grand sceau en cire jaune (V. M. de Saint-Mery, Description topographique... de S. Domingue, (rééd.) Paris, 1958 t. I, 74) - idem, Commission de M. de Tracy, gouverneur-lieutenan general, donnée à Paris le 19 9embre lan de grasce 1663 et de nostre reigne le sixiesme, Lectre signée Louiset sur le rabat Per regem, de Lionne (secrétaire d'Etat).

${ }^{14}$ R. P. Raymond Breton, Relations latines (B), 1656, $158:$ : De statu et numero Gallorù̀ in insula : Coeterum in insula Guadalupa hodie nulli, ut dictum est, morantur indi ; etsi ad commercia frequentes veniant. Gallorum autem catholicorum supra duodecim millia numerantur. Nigritarum ( qui Africae populi sunt et mancipiorum more et potius bestiarum ad omnia servilia opera emuntur a suis regibus; et 1500 aut 2000 libris tabaci sani et robusti sunt et juvenes ab Anglis aut Hollandis venduntur; gens pia, docilis, fidelis, et vere ad servendium nata, laboriasa, parvo cotenta) utriusque sexus ad tria et amplius millia, qui omnes fere a nostris in fide instructi et baptisatti eo facilius, quo a baptismo daemonibus amplius non vexentur et sapulent et saepius ante contingebat. »- Sur les travaux préparatoires du Code Noir : projet de 1683, C.H.A.N. F 90, titre : de la liberté, § 4.
} 
Cet état de chose ne peut perdurer ${ }^{15}$. Il faut limiter et borner cet esclavage des temps nouveaux. Ce sera l'objet du Code Noir Louis.

Mais pourquoi ce «Code»? Son objet ne pose apparemment pas de problème : il réglemente la situation de la classe servile africaine de façon maladroite et selon la dure mentalité du temps. Il voit dans la personne de l'esclave un simple objet tant qu'il n'est pas baptisé. Son statut mobilier est illogique et anachronique ${ }^{16}$. Plus tard, précise E. Géraud-Llorca ${ }^{17}$, on le qualifiera de bien immeuble par destination, ce qui constituera une protection de ce dernier. Surtout, les juristes n'ont plus en mémoire le fait que l'esclave à Rome n'est ni meuble ni immeuble, qu'il est simplement assujetti à la puissance du maître (dominica potestas) car res mancipi. C'est pour cette raison qu'il peut être par la suite émancipé ${ }^{18}$ à l'instar du fils de famille. Le Code Noir s'intéresse dès ses premières dispositions (art. IV) au régime même de l'administration du travail sur l'habitation car ne "seront préposez aulcuns commandeurs a la direction des nègres, quy ne fasse profession de la religion catholicque... a peine de confiscation desditcts nègres contre les maistres quy les auront préposez et de punition arbitraire contre les commandeurs quy auront accepte ladicte direction ». Le principe de catholicité trouve application jusque dans l'exploitation et le régime économique de l'habitation. L'article $\mathrm{V}$ s'adresse formellement à ceulx de la religion prétendument réformée. L'article VI vise le respect du repos hebdomadaire et du chômage les jours de fêtes religieuses nombreuses dans l'année liturgique d'Ancien Régime.

Mais ces quelques lignes n'ont répondu que partiellement à la

\footnotetext{
${ }^{15}$ Le jésuite Mongin fait état à St Christophe de conduites très curieuses : quelques maitres « sont allez à des excès que n'ayant que des negresses, ilz ont payé des neggres estrangers comme des étalons pour en avoyr les enfans qui sont toujours au maistre de la mère... (registre Mongin, in Bibl. Carcassonne VII, 112.) ». La pratique semble minoritaire, elle heurte semble-t-il les religieux.

${ }^{16}$ Illogique, car dans l'Antiquité - et surtout à Rome - l'esclave est attaché à la famille du maître (familia rustica ou urbana); anachronique, car la reprise des solutions romaines a été mal comprise d'où la qualification surannée de res appliquée à l'esclave d'Amérique. La coutume règlera ce point en faisant de l'esclave une chose immeuble par destination, ce qui déjà paraît fort différent. Surtout, on oublie que certains juristes romains, dont Gaius, situent les esclaves dans leurs oeuvres non pas parmi les choses, mais parmi les personnes (Institutes, Liv. I, §§ 52 - 54).

${ }^{17}$ La coutume de Paris-Outre-Mer : l'habitation sous l'Ancien Régime, R.H.D. vol. 60, Sirey, spéc. 247 qui porte interdiction de la saisie des esclaves de culture attachés au sol en tant que pars fundi.

${ }^{18}$ V. notre étude : Le droit romain aux Antilles : l'affranchissement in B.S.H.G. $2000(=$ R. H. D. 2001 vol. 79, 347-360).
} 
question Pourquoi le Code ? La raison d'être de celui-ci repose sur la règle fondamentale de la catholicité. L'esclave, brute épaisse venue d'Afrique ? L'esclave, animal humain dénué de toute âme ${ }^{19}$ ? L'esclave, matériel voué au travail et chargé de se reproduire ? L'esclave, simple catégorie d'individus ne disposant ni de droit ni d'une personnalité même réduite ? Non, tout cela est impossible. Le roi et le marquis de Seignelay l'ont fort bien compris. L'esclave doit être instruit dans la religion catholique et baptisé. Les maîtres ont à son égard des obligations «civiles » telles que le nourrir, l'habiller, le soigner, lui donner une sépulture décente, c'est-à-dire religieuse. Mieux encore : l'esclave, par ses mérites, peut être affranchi et devenir à son tour un régnicole, sujet de droit, certes minuto iure - mais non simple objet passif ! du roi. Sur tous ces points nous renvoyons à des études déjà publiées ${ }^{20}$. Paradoxalement la première mission du Code Noir, avant même d'établir et de fixer des règles de servitude, consiste à prendre en compte l'aspect humain de l'individu, même s'il est en captivité loin du royaume. C'est dans ce même état d'esprit qu'intervient l'Ordonnance concernant la police des Nègres et gens de couleur Libres du 25 décembre $1783^{21}$ précisant dans son Préambule que «le Gouvernement doit veiller sur le sort de ces hommes que la loy impérieuse du besoin peut faire, par cette raison, sortir de leur devoir. Il convient en mesme tems que l'on contiendra l'esclave, de veiller a ce que le maître remplisse un engagement que l'humanité luy a fait contracter avec la loy en l'obligeant a vettir et nourrir son esclave, et a le traicter humainement $(\ldots)^{22} »$. Certes, il ne convient pas de verser dans un angélisme stupide et béat mais de reconnaître que c'est la règle fondamentale de catholicité de la Couronne qui posait ici des contraintes qui ne sont explicables de facto et de jure que si l'on fait expresse mention de la nature constitutionnelle (Ph. Sueur) des Loix du royaulme sous l'Ancien Régime. Cette norme trouve une curieuse application dans l'histoire de la colonisation française Outre-Mer.

${ }^{19}$ V. Seneque (fils), Entretiens - Lettres à Lucilius chez R. Laffont, éd. établie par Paul Veyne, Paris, 1999, 1094 ; V égal. texte de P. Grimal dans Sénèque et la conscience de l'Empire spéc. Epist. XLVII, Lettres à Lucilius (circa, 64 de n. è.) spéc. 315 sq.

${ }^{20}$ not. «Droit romain aux Antilles : la pratique des affranchissements », in B.S.H G. $\mathrm{n}^{\circ} 121$ 122, 1999, spéc. 33-74 - « Droit romain et servitude aux Antilles », B.S.H.G. n 127-128, $2001,25-46 \ldots$

${ }^{21}$ dénommé Code Noir G, comme provenant du Gouvernement local, code publié en 1784 dont les auteurs sont les Vte de Damas, general et J. Petits, Sr de Viévigne, intendant.

${ }^{22}$ Curieusement il n'y a pas dans le préambule de l'Ord. mention faite à la religion : est-ce là un signe de l'avancée des idées philosophiques et des Lumières aux Isles d'Amérique ? 
Pour toutes ces raisons l'expansion française dans les contrées d'Amérique a été en principe soumise à des Administrateurs généraux (de la Marine, en un premier temps, du cadre général par la suite, après 1761) mais aussi surveillée et contrôlée plus ou moins directement, de manière officieuse, par divers ordres religieux dont le statut général n'a jamais été clairement défini.

Le statut des clercs et religieux des Antilles est plus que flou, il n'existe quasiment pas. Les lettres de commissions des Administrateurs généraux n'y font même pas allusion ${ }^{23}$. Les ministres du culte dépendent d'un père supérieur à l'échelon local parfois qualifié de préfet, plus rarement de vicaire dont on ne cerne pas toujours aisément les compétences hormis celles de la liturgie et de la discipline interne. De façon précise, on sait que les religieux, carmes et capucins par exemple, sont soumis à l'appréciation d'un père visiteur voire du supérieur ou d'un provincial ${ }^{24}$. Il en va sûrement de même pour les jésuites et les dominicains ${ }^{25}$. Le P. Labat fait allusion à une éventuelle saisine de l'intendant en matière de discipline sans préciser davantage $^{26}$. A défaut d'évêque les différents ordres demeuraient sous le contrôle pratique de ce personnage. Le conseil souverain ou supérieur, sur réquisition de son procureur général, refusait parfois d'enregistrer des lettres d'accréditation des préfets apostoliques ${ }^{27}$; c'est là une de ses rares compétences en matière religieuse ${ }^{28}$. Il $\mathrm{y}$ a là une certaine application des

${ }^{23}$ ou alors si vaguement...

${ }^{24}$ Ainsi le 5 mars 1726 le Secr. d'Etat écrit à l'intendant Blondel : "J'ai veü la copie de la lettre escrite par le superieur des Carmes de la Guadeloupe a M. de Feuquieres sur la pretention du Sr de Chamoy que le pain benit soit presenté aux officiers du Conseil.... » Ailleurs, il est question du P. Provincial de Normandie (ordre des capucins) auquel les Antilles sont rattachées. Il revient d'une mission de surveillance en 1751 dans îles-du-Vent. Ce dernier se plaint ouvertement affirmant «que les exercices de la religion sont assez négligés et que les religieux qui veulent travailler avec un certain zèle à y ramener leurs paroissiens sont souvent exposés à forts desagremens dont leur estat devroit les garantir. »

${ }^{25}$ i.e.: Lettre du P. jésuite Mongin à son supérieur en 1679 : sur le sujet :V. B.S.H.G. 1984, ${ }^{\circ}$ 61-62, art. sur L'évangélisation des esclaves au XVII s. - Lettres du RP. Mongin, (lettres présentées par M. Chatillon).

${ }^{26}$ Voyages aux isles, coll. Phébus-Libretto, présentation M. Le Bris, Paris, 1993, spéc. 191, 203...

${ }^{27}$ Cela soulève la question de la validité des lettres d'attaches qui ne sont adressées qu'aux préfets apostoliques parce qu'elles émanent d'une autorité étrangère (cf. Lettres patentes de juill. 1763, art. 5, portant sur la dispense de lettres d'attaches pour les supérieurs et vicaires généraux).

${ }^{28}$ L'appel comme d'abus n'est guère applicable aux îles d'Amérique. L'affaire du P. Sigismond, refusant d'administrer la communion à une demoiselle Truguet et entraînant un 
idées gallicanes dans les îles d'Amérique, véritable observatoire, ce qui explique l'absence d'official ou juge d'Eglise ${ }^{29}$. Or, cette situation perdurera jusqu'à la fin du XVIII' ${ }^{\mathrm{e}}$ siècle $^{30}$.

La question de la richesse des prêtres et ordres religieux aux Petites Antilles a sans doute fait couler beaucoup d'encre. Il paraît établi en effet qu'une bonne partie du clergé vivait à la façon des habitants et était installée dans des domaines où l'on produisait du sucre ou du tafia... ${ }^{31}$. En revanche les capucins n'ont jamais eu de biens en Guadeloupe, ni en Martinique. Les frères de la charité ne disposent que d'une moitié d'habitation ${ }^{32}$. Surtout, la législation royale intervient par le Bureau des colonies pour surveiller ce genre d'exploitation et interdire toute acquisition par des religieux sans permission expresse des Administrateurs sous peine de voir de tels biens réunis au Domaine ${ }^{33}$.

Les historiens ont mis en évidence la grande influence des ecclésiastiques en terres coloniales malgré la «disette de prêtres séculiers » (Peyronnin) aux isles au XVII ${ }^{\mathrm{e} 34}$ comme au $\mathrm{XVIII}^{\mathrm{e}}$ siècle $^{35}$. A l'inverse

scandale avec le sire de Châteaubrun (Ste Anne de Guadeloupe, 1757) le prouve. Le père capucin est renvoyé sur ordre en Normandie (C.H.A.N. B105 fo 47, 3 sept.). Dans les faits les questions religieuses (discipline, approche et dispense des sacrements) ne regardent que l'intendant et non point les membres du conseil souverain de la Martinique).

${ }^{29}$ Ce vide canonique empêche ainsi toute contestation, tout scandale ou frais inutiles, C.H.A.N. B65 (2) fo 310, arr. du Conseil d'Etat confirmant une décision du Cons. souv. de Martinique faisant application de l'art. 34 de l'édit de 1695 concernant les sacrements et la discipline ecclésiastique. Sur la quest. de répartition des compétences entre clercs et laïcs : F. -X. Emmanuelli, Etat et pouvoir dans la France des XVI et XVII s., Paris, Nathan-Univ, 1992, 134 ss.

${ }^{30}$ En 1773, toutefois, le roi jugea convenable d'envoyer à Saint-Domingue un évêque in partibus, Mgr Roque, C.H.A.N. B145 fo 32.

${ }^{31}$ C.H.A.N. C8A 27, $\mathrm{f}^{\circ} 10-12$ : les jésuites de Saint Pierre disposent de plus de 150 nègres et réaliseraient un revenu de $34000 \mathrm{lv}$. par an. Ils toucheraient de plus $20000 \mathrm{lv}$ de rente. On dit les PP. carmes très riches.

${ }^{32}$ Soit environ 135000 lv. et 60 nègres de culture et de maison (C.H.A.N. C8A 27, fo 13 ).

${ }^{33}$ Cf. Lettres patentes d'août 1772, C. M., t. 1, 158 ; voir position de l'intendant Blondel affirmant que les biens "leur [les religieux] permettent de ne pas dépendre de leurs appoinctemens. Ils sont a la tête d'une richesse considérable et qu'il faut les empescher de s'aggraver davantage... » (C.H.A.N. C8A $\left.34 \mathrm{f}^{\circ} 319\right)$.

${ }^{34}$ On compte à peine une centaine de prêtres et de religieuses aux Antilles en 1687, soixante dix sept églises contre plus de quatre cents sucreries et deux cents indigoteries. Il y a donc des difficultés dès les origines à fixer clercs et religieux dans les possessions d'Amérique, qu'elles soient tropicales ou de Nouvelle France... 
l'Administration royale a dû faire appel à différents ordres religieux pour assurer l'évangélisation et les exercices habituels de la religion. En somme, ces congrégations jouissent quasiment du monopole de la Cura animarum, à savoir du soin des âmes en général et du respect de l'orthodoxie en particulier - t plus spécialement - de la cura servorum, le souci des esclaves.

Le soin des âmes s'entend précisément du soin porté par les clercs séculiers ou réguliers aux intérêts cultuels de la population et à l'érection de paroisses administrées par des fabriques ${ }^{36}$. De manière générale cette action se traduit de plusieurs façons : les religieux exercent une mission de maintien de l'orthodoxie mais également contrôlent la moralité de leurs paroissiens ${ }^{37}$. Ils administrent les sacrements ${ }^{38}$. Ils se préoccupent aussi du for externe. A ce titre ils se montrent très opposés aux fausses croyances, déviances, hérésies ${ }^{39}$; par ailleurs ils combattent les superstitions et les pratiques occultes perverses notamment transmises par les Noirs déportés d'Afrique. Divers témoignages font état de magie ou d'ensorcellement ${ }^{40}$. L'Eglise locale, sur ce point, ne peut qu'appliquer les principes de droit positif religieux et d'ordre public applicables dans le reste du royaume. Autre domaine d'intervention : les autorités religieuses influencent le comportement des magistrats comme des particuliers. Les curés rendent publiques des décisions au prône de la messe ; au plan du for interne, autrement dit de l'individu, ils confessent les croyants et accordent l'extrêmeonction. Ce point, certes, fort intéressant, nous concerne ici beaucoup moins ${ }^{41}$.

\footnotetext{
${ }^{35}$ On note par ex. en 17639 dominicains curés payés à 360 lv, 7 dominicains vicaires à 270 lv, 18 missionnaires capucins à $422 \mathrm{lv}$, un aumônier cordelier à $1500 \mathrm{lv}$, un aumônier à SaintChristophe , 1500 lv, un autre à Saint-Eustache, 1300 lv : V. corresp. des Administrateurs Damas et Foulquier, 8 mai 1787, A. N., C8A 67, f 9, n 24 (état estimatoire).

${ }^{36}$ Ces divers conseils de fabrique disposent de fonds alimentés par des amendes prononcées par le subdélégué ou le commis à la police (C.H.A.N. B49 (1) fo 301 : miss. Secr. d'Et. à Blondel ).

${ }^{37}$ Selon L. R. Abenon, ouvr. cit., t. II, 151, le prêtre « doit aussi, contre vents et marées, se faire le gardien d'une certaine tradition morale. »Certains curés dénoncent les débordements sexuels de mulâtresses et de jeunes gens dits de bonne famille. Ils vont jusqu'à chasser des paroissiennes dont la conduite les rend indésirables. Les huissiers s'en mêlent parfois. Des affaires scandaleuses sont parfois portées à la connaissance des gouverneurs (aff. Marianne Labourde, C.H.A.N. F3 $224 \mathrm{ff}^{\circ} 327$ à 355).

${ }^{38} \mathrm{Au}$ for interne, ils interviennent par la pratique de la confession qu'ils essayent d'encrer dans les moeurs coloniales.

${ }^{39}$ A ce titre les religieux doivent garder pur le depositum fidei qui est par nature involutif $(\mathrm{O}$. Clément) depuis le concile véritablement œcuménique de Nicée au début du IVe siècle.

${ }^{40}$ Statuettes, bâtons qui parlent, pluies miraculeuses etc. V. P. LABAT, ouvr. cit., 114 - 118.

${ }^{41}$ Il n'y a pas trace de confession publique sauf en matière criminelle : amende honorable, cf.
} 
L'Eglise catholique aux Antilles a eu très tôt à se préoccuper du sort des Juifs et des protestants. Il y va du respect du droit public politique et religieux. Or, cet équilibre fut-il maintenu au prix de persécutions ordonnées par les milieux ecclésiastiques ? Comme le prétend L. R. Abenon il ne semble pas que la persécution - si elle a existé - ait été le fait des prêtres ${ }^{42}$. Bien au contraire. Avant l'existence de rapports dressés par les religieux, on a pu noter ceux des Administrateurs généraux qui se plaignent jalousement du trop grand nombre et de l'essor économique des Juifs d'Outre $\mathrm{Mer}^{43}$. Il convenait donc d'observer dans les colonies lointaines le même ordre public religieux qu'en France. Par ailleurs, nous ne trouvons pas trace d'une véritable persécution ; tout au plus peut-on parler de ségrégation religieuse. Et cela est encore fort discutable à l'égard des juifs en raison même d'une instruction du Secrétaire d'Etat permettant leur libre exercice cultuel à titre privé $^{44}$. En effet, on sait que prêtres et religieux ne désiraient pas de conversions forcées, ce que confirme le $\mathrm{P}$. Labat, et ce, en raison du risque d'apostasie. Les cas d'abjurations forcées ne sont pas nombreux : à la limite ils s'expliquent parfois pour des raisons financières (recouvrer par exemple une partie du patrimoine familial à la suite de faillites frauduleuses ${ }^{45}$ ). Nous disposons d'une espèce où un certain P. Mane, curé de Capesterre en 1726, n'hésita pas à entreprendre la conversion d'une fille de la bourgeoisie locale in articulo mortis ${ }^{46}$. Dès le XVII ${ }^{\mathrm{e}}$ siècle une missive royale de Louis XIV du 23 mai 1671 adressée au lieu tenant-general de Baas (en fait lire : de Batz), qui fut protestant à l'origine, lui enjoint «de tenir la main a ce que les Juifs jouissent des mesmes privilesges que les autres $[\mathrm{h}]$ abitans et leur laysser une

carton F5 A 28 pour la Martinique.

${ }^{42}$ Ouvr. cit., t. II, 151. - En fait, il semble que l'expulsion des Juifs de Martinique, en 1683, ne fut que temporaire.

${ }^{43}$ C.H.A.N. C8 A3 f 50 : miss. des Adm. 10 févr. 1681 : «Je croys que le roy doit faire un reglement pour les Juifs. Ils possedent des terres, des esclaves chrestiens et des commis et ils se multiplient beaucoup, de sorte que le commerce est presque tenu par eulx. » $\mathrm{Cf}$. art. I du futur «C.N.L. ».

${ }^{44}$ C.H.A.N. C8 B4, instruction du $1^{\text {er }}$ mai 1682 adressée au Sr Bégon. Les dispositions ultérieures de la monarchie paraissent adoucir l'art. $\mathrm{l}^{\mathrm{er}} \mathrm{du} C . N$. : " enjoignons à tous noz officiers de chasser hors de noz isles tous les Juifs qui y ont establys leur résidence. » Cette ernière disposition était contemporaine de l'Edit de Fontainebleau...

${ }^{45} \mathrm{C}$ 'est le cas pour un certain Isaac Duquerry qui est à l'origine d'une procédure longue et coûteuse (C.H.A.N. col. F3 226, $\mathrm{f}^{\circ}$ 181, acte d'abjuration entre les mains du duc de Gesvres, 5 mars 1745).

${ }^{46}$ C.H.A.N. C8A35 f ${ }^{\circ} 51$ (Mlle Godet du Bois refuse l'extrême-onction). 
entière liberte de conscience ${ }^{47}$. » Au XVIII ${ }^{\mathrm{e}}$ siècle, les idées de l'Aufklärung catholique se répandent ${ }^{48}$; on revient à une tolérance plus affirmée. C'est ainsi que l'instruction aux Administrateurs de Choiseul concernant la colonisation en Guyan[n]e recommande par deux fois « de ne pas inquiéter les non-catholiques et de leur laisser exercer librement leur culte ${ }^{49}$. "

La cure des esclaves est donnée aux religieux dès 1664, c'est-à-dire au commencement de l'importation des populations africaines aux Antilles françaises. Cette mission très particulière se voit renouvelée par l'ordonnance du 14 septembre 1672. Elle permet expressément aux religieux et missionnaires de tous ordres de visiter les esclaves... soit aux champs ou jardin, soit ès cazes ou ailleurs... où ils se présentent pour les instruire en général ou en particulier. De ce fait les religieux sont bien placés pour signaler aux autorités civiles les mauvaises habitations, celles où règne soit un laxisme effréné engendrant des querelles entre nègres d'habitations voisines soit une violence répressive atroce des maitres. Il y a tout lieu de croire que les habitations mal tenues sont dénoncées et les propriétaires montrés du doigt car ceux-ci mettent l'équilibre social et la tranquillité publique en péril ${ }^{50} .1664$ est précisément l'année où s'installent les jésuites, membres de la Compagnie de Jésus. Il est d'ailleurs très probable que ces jésuites obtinrent le quasi-monopole de l'évangélisation des esclaves en raison de leur nombre ${ }^{51}$ dans les colonies mais aussi de la politique

\footnotetext{
${ }^{47}$ V. aussi Instructions des 25 avr. 1687 et 1 er sept. 1688 "recommandant de ne point mollester les subjets de la religion prestendüe reformée \& de s'efforcer de les tenir dans les collonyes » (V. Em. Petit, ouvr. cit., t. II, 429).

${ }^{48}$ V. Abbé de Very, Journal, de Witte, Paris, s.d., 382 ss. (politique en octobre-décembre 1775 au sein du Conseil du roi).

${ }^{49}$ M. Pauliat, La politique commerciale de l'Ancien Régime, 1887, 106 ss.

${ }^{50}$ V. aff. Cartier, arr. Cons. souv. Mart., 9 janv. 1727, C H. A. N. B50, 264 - aff. Marthe Roblot, Cons. souv. Mart., oct. 1736, C. H A. N. col. B64, 3O3 - deux arr. Cons. souv. Mart. cassés en Cons. d'Etat (janv. 1740) provoque une décision ministérielle ordonnant instruction complémentaire sur les cruautés excès et sévices subis par une esclave (miss. 7 août $1740, \mathrm{C}$. H A. N. col. F3 256, 993-994) - Sr Langlois, arr. Cons. sup. Guad., 4 nov. 1743, C.H.A.N. F3 226 , 65 etc... Dans la plupart des cas l'habitant est déclaré incapable de posséder des esclaves pour l'avenir, il est donc « ruiné » et doit quitter les lieux après avoir payé amende : parfois le Secr. d'Etat commande de fayre exécuter l'arrest en toute rigueur sans entrer en consideration du peu de faculté (sic) du particulier qui ne mérite aucune grasce.

${ }^{51}$ Nombre qui augmente sensiblement : ainsi on peut noter en $1685: 11$ membres de la Société de Jésus, 6 à Saint-Christophe, 3 en Guadeloupe ; en 1705 il y a 24 jésuites pour l'Amérique méridionale, pouis 35 en $1714 ; 40$ en 1723 en encore 60 en 1737.
} 
de l'Ordre ${ }^{52}$. Cette attribution «leur permettait d'intervenir dans la vie de la colonie sous prétexte de défendre «leurs ouailles ${ }^{53} »$. Les jésuites ne sont d'ailleurs pas les seuls clercs gênants aux isles. J. Petitjean-Roget rapporte les propos de l'ordonnateur principal Mithon précisant que «le supérieur des Jacobins a changé de conduicte depuis le départ du père» (Labat) et d'ajouter : «ce qui prouve que le P. Labat dont l'esprit est remuant et passionné l'entretenoit dans le mesme esprit (sic). Il est très prudent de l'empescher de retourner aux isles. Il ne pourroit qu'y causer beaucoup de désordres ${ }^{54}$. ».

A la vérité on peut se demander si la présence des religieux est jugée comme toujours très souhaitable dans les possessions françaises d'Amérique. On prétend même qu'elle serait source de difficultés et d'intrigues. Et une certaine méfiance s'est fait jour assez rapidement. Notamment, on ne désire pas la présence d'évêque dans les Petites Antilles françaises malgré des hésitations de Versailles ${ }^{55}$. Mieux encore, les jésuites organisent dès le début du XVIII ${ }^{\mathrm{e}}$ siècle des confréries d'esclaves, initiatives confirmées en Cour de Rome l'année $1745^{56}$.

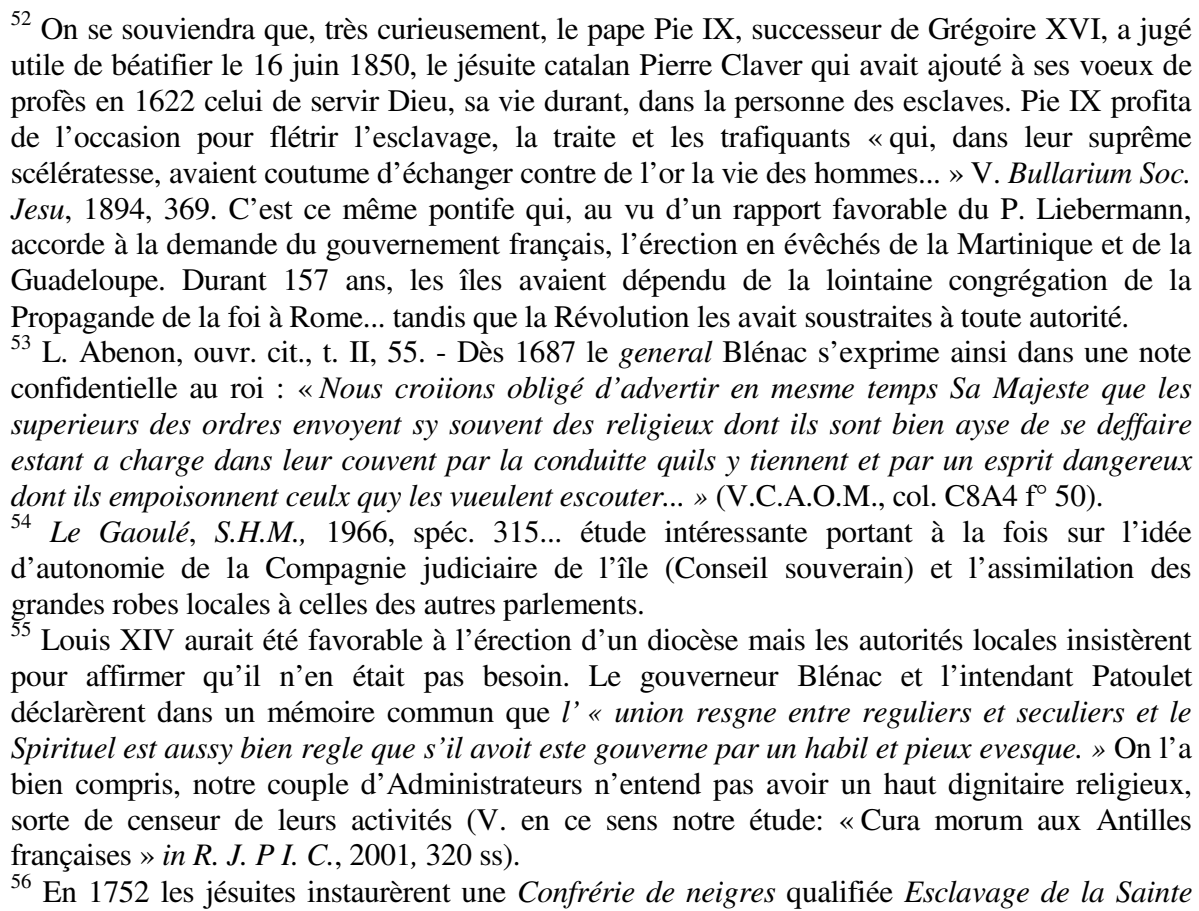


La cure des esclaves s'exprime donc de différentes façons : visite des nègres de culture, enseignement, retraites en différents points de l'île, à diverses époques (Carême, Toussaint) ${ }^{57}$. L'organisation des temps de prières est sévèrement critiquée par les maîtres, parfois même, par les autorités. Surtout, certains ordres - et précisément les jésuites, propriétaires d'esclaves - montrent que leurs « abitations » sont mieux gérées parce que plus humaines ${ }^{58}$. Il n'en demeure pas moins que les religieux semblent avoir une politique qui se traduit de plusieurs manières : l'une consiste à établir, en tant qu'officiers royaux, les actes baptistaires, une autre, à enseigner voire à se démarquer des Autorités, enfin une dernière qui relève de la mission traditionnelle d'aide et d'assistance.

Par la pratique des actes de baptême, les religieux sont seuls habilités à livrer des actes d'état-civil. Ils en ont le monopole ici comme dans tout le reste du royaume en vertu de l'édit de Villers-Cotterêt de 1539. En application de cette règle d'ordre public, ils confectionnent des actes qui constituent de véritables titres authentiques. Il arrive assez fréquemment que des clercs, selon A. Lebeau ${ }^{59}$, modifient la réalité des filiations et déclarent Libres des enfants en servitude ou dont les parents ne sont pas manifestement affranchis. La problématique n'est pas ici nouvelle : déjà les pères capucins de l'île de $S^{t}$. Christophe, en 1652, tenaient qu'après le baptême les enfants devaient être affranchis et que c'estoit choze indigne de

Vierge dans leurs paroisses de Guadeloupe en vertu d'un bref romain. Il y eût selon Abenon une vive résistance des autorités (ouvr. cit., t. II, 59).

${ }^{57}$ Les retraites du P. supérieur Dubois s.j., des PP. Casimir et Desbouges en Guadeloupe, sont extrêmement critiquées et font l'objet de plaintes (C.H.A.N. F3 71, 65, 66ss - « curés des nègres $\gg$ ou catéchistes de fortune, 70).

58 Certes, à l'exception des capucins qui ont fait voeu de complète pauvreté, les religieux des Petites Antilles, et plus particulièrement les jésuites, sont propriétaires d'esclaves. Certaines habitations ont fait l'objet d'études (i..e. Bisdary sur la paroisse de Dos d'âne, Basse-Terre, actuellement Gourbeyre, V. J. Faloppe, Résistance d'esclaves et ajustement au système : le cas de la Guadeloupe dans la première moitié du XIX ${ }^{e}$ s, B.S.H.G. n68, 1986, 31 - 52, spéc. 44 sur l'historique du pécule au XVIII ${ }^{\mathrm{e}} \mathrm{s}$. dans les habitations bien tenues malgré l'interdiction de l'art. 28 C. N. ce pécule leur permettant le rachat - A. Gautier, Les esclaves de l'habitation Bisdary, 1763 - 1817, B.S.H.G., $\mathrm{n}^{\circ}$ 60, 1984, 13ss : ancienne habitation des jésuites, vendues en 1766 : l'auteur déclare «que les esclaves de Bisdary contredisent par bien des aspects les images habituelles... beaucoup sont qualifiés, ils sont inclus dans des réseaux de parenté, ils vivent avec leur père et mère puis en couple et ont de nombreux enfants... les jésuites ont mené une politique de mariage originale puisque seuls $10 \%$ des esclaves guadeloupéens sont mariés »).

${ }^{59}$ La condition des affranchis, th. Dr. Poitiers, 1902, 64 (citant ord. 29 déc. 1774, v. infra n. 62). 
se servir de son frère chrestien comme esclave (... $)^{60}$. Une ordonnance du 15 juin 1736 essaye déjà de contrer la politique des religieux ${ }^{61}$; de plus, selon une jurisprudence constante à la fin du XVIII ${ }^{\mathrm{e}}$ siècle, les curés se voient reprocher d'insérer la filiation du père alors que l'enfant est né « naturel ${ }^{62} »$. Une ordonnance des Administrateurs du 19 décembre 1774 poursuit cette politique en vue d'instaurer une sorte de procédure de vérification des actes baptistaires ou notariés par l'administration du Domaine ${ }^{63}$. On s'est relativement peu posé la question d'en savoir l'exacte raison qui semble fort simple. Conscient des dégâts du système esclavagiste au plan de la parenté, certains prêtres n'hésitent pas à fixer dans leurs actes une filiation paternelle afin d'impliquer les pères dans le processus de responsabilité lié à la génération humaine. Il leur arrive aussi de donner le nom du père naturel à l'enfant issu de cette union illégitime. La réaction des autorités civiles sera vive. Les Conseils souverain ou supérieur formulent par la voix de leurs procureurs généraux respectifs des reproches très sévères aux ecclésiastiques

${ }^{60}$ V. Maurille de Saint-Michel, Voyage des isles Camercanes, 1652, 81-85.

${ }^{61}$ Cf. Ord. 15 juin 1736 in Dessalles, ouvr. préc. t. I, 375. Des sanctions sont prévues : confiscations de la mère et de l'enfant au maître, amendes contre les religieux... peines en fait inappliquées ou inapplicables... V. C. H. A. N., F3 134, f 176).

${ }^{62}$ Cf. arr. Cour sup. de Guad., 15 nov. 1763, C.H.A.N. col. F 227, C. G., 277 : art. 2 intime expressément aux curés de ne donner aux mulâtres et affranchis que le nom de leurs pères légitimes dans l'acte de baptême. Ainsi dans le cas ou un père blanc et une mère de couleur seraient unis en légitime mariage, l'enfant devait naturellement porter le nom de son père. Le Cons. sup. de Port-au-Prince, 16 juill. 1773, reprend dans son Préambule les mêmes motifs dans un arrêt de règlement au sujet des négresses, mulâtresses, quarteronnes et mestives non mariées qui feraient baptiser leurs enfants : le nom de baptême et un surnom tiré de l'idiome africain ou de leur métier ou de leur couleur doivent suffir. La peine portée est considérable car elle est fixée à 1000 lv. d'amende sans compter les dommages et intérêts et la réparation civile de l'Ancien droit.

${ }^{63}$ Ord. préc., art. 10, 11, 12 s'adressant aux curés et religieux, aux notaires et greffiers, rappellant les dispositions classiques de l'ancienne réglementation (ord. de 1713 et 1736) restées jusque là sans trop d'effets (...) ; il est enjoint aux personnes susdésignées de fournir annuellement au directeur général du Domaine un estat certifié par eulx de tous les enfans de couleur qu'ils avoyent battisés sous la qualitte de libres... afin que l'on vérifie s'ils estoient vrayment nés libres... (C.H.A.N. F $261-=C$. M. $559-567$ ). La présente ordonnance, jugée dangereuse pour les gens de couleur libres qui peuvent voir leur possession d'état remise en cause ad nutum a été cassée en Cons. d'Etat du roi le 18 août 1775 (cf. P. Fr.-R. Dessalles, Annales du Cons. souv. de la Martinique, (t. II) Bergerac, 1786, Paris, rééd. L'Harmattan, 1995, annoté par B. Vonglis. 
et fixent même des peines (amendes) voire des blâmes ${ }^{64}$. En attendant rien n'y fait. Les mesures de justice ne peuvent en ce domaine être rétroactives. En général, elles n'invalident jamais de tels actes (...) Tout cela revient à dire que les mulâtres et autres gens de couleur ingénus se faisaient appeler ordinairement du nom de leurs pères naturels de race blanche d'une part, et que, d'autre part, les affranchis prenaient coutumièrement le nom du maître qui leur avait donné la liberté, suivant en cela la tradition romaine ${ }^{65}$.

Autre point à soulever ici. Il s'agit du comportement des religieux et notamment des jésuites à l'endroit des méfaits de l'institution esclavagiste. Certes, on a pu être déçu par une attitude jugée trop timide voire timorée de ces derniers à l'égard de l'esclavage ${ }^{66}$. Quoi qu'il en soit l'histoire en général $^{67}$ et les travaux récents en particulier tendent à démontrer que les jésuites sont en quelque sorte ceux qui ont le plus revendiqué cette cura des esclaves $^{68}$. Toutefois il semble que de nombreux religieux aient fait l'objet

\footnotetext{
${ }^{64}$ On trouve dans cette réglementation une ord. des Administrateurs de la Martinique donnée tardivement le 6 janv. 1773 (V. Durand-Molard, ouvr. cit., t. III) interdisant dorénavant (...) aux negres et autres gens de couleur libres de porter les noms de familles blanches de la colonie. - Cf. ord. 15 juin 1736 (= Dessalles, Annales... I, 375ss.). A vrai dire ces textes successifs (ord., arrêts... ) semblent indiquer qu'ils sont très mal exécutés.

${ }^{65}$ En ce sens, A. Lebeau, ouvr. cit. 87. - Y. Debbasch, Couleur et liberté, le jeu du critère ethnique dans un ordre juridique esclavagiste... t. I., L'affr. dans les possessions françaises d'Amérique, Paris, Dalloz, 1967, 70ss - L. Peytraud, L'esclavage aux Antilles françaises avant 1789, d'après des documents inédits des archives nationales, t. 1, 1897. A ce jour il manque une étude sur l'onomastique antillaise qui porterait sur les origines, les coutumes de fixation et de dévolution des noms, ainsi que sur l'histoire du contentieux du nom sous l'Ancien Régime et le début de l'ère républicaine (avant et après 1848).

${ }^{66}$ Voir en ce sens les travaux classiques du P. A. Gisler et récemm. de Ph. Peyronnin, art. portant sur les Contours d'une pastorale ultramarine. Les missionnaires jésuites dans les sociétés d'habitation des Antilles et Guyane françaises_aux XVII et XVIII siècles, in B. S. H. G., $\mathrm{n}^{\circ} 126,2000$, V spéc. 32 - 33 et in fine une conclusion assez pessimiste et sévère sur l'action des jésuites, 68.

${ }^{67}$ G. Debien rappelle «que les esclaves regardaient les jésuites comme leurs protecteurs, et les jésuites se regardaient comme leurs défenseurs... » (V. Les esclaves aux Antilles françaises XVII - XVIII $I^{e}$ siècles, Basse-Terre - Fort-de-France, 1974, 285). De même, P. Pluchon remarque qu'à Saint-Domingue les jésuites ont été fort convaincus de leur «mission tutélaire » et qu'ils l'ont accomplie «avec fermeté »: Histoire de la colonisation française, Fayard, Paris, 1991, 427. On va jusqu'à citer l'oeuvre du P. V. Filutius, Quest. morales, t. II, jugée sulfureuse parce qu'approuvant la désertion ou les voleries de l'esclave maltraité.

${ }^{68}$ Pour s'en convaincre : cf. Epistulae critiques des PP. Pelleprat (rudesse de l'esclavage) et Mongin, à leurs supérieurs, préc.
} 
de procédures de renvoi rondement menées soit en raison de fautes personnelles dans l'exercice de leurs ministères ${ }^{69}$ soit de leurs convictions trop avancées ${ }^{70}$; d'autres s'expriment trop librement et réagissent à des condamnations brutales; quelques uns sont emprisonnés ${ }^{71}$; d'autres encore célèbrent avec ornements rouges (pour les martyrs !) des offices pour les nègres suppliciés et leurs donnent sépulture décente ${ }^{72}$. Lors d'affaires retentissantes des procureurs généraux livrent leurs opinions et pensent par exemple que les jésuites vont jusqu'à troubler l'ordre public colonial en " attirant les nègres plus a eux qu'a la religion ${ }^{73}$... d'excuser le marronnage, d'autoriser le vol des denrées alimentaires, de les détourner du travail à force de prières et de retraites, de les instruire hors la présence des maîtres... de vouloir les séparer des autres fidèles... ». C'est sans doute sur le fondement de ce genre de réquisitoire qu'il faut chercher les véritables raisons de la suppression de la Compagnie de Jésus dans le royaume mais également et surtout dans les colonies ${ }^{74}$.

\footnotetext{
${ }^{69}$ Hormis le cas de l'abbé du Lion, fils du gouverneur du même nom, pour son inconduite notoire il est un certain nombre de religieux dont la présence a paru indésirable : cas d'une décision rendue par un juge inférieur de Martinique prononçant la bannissement d'un prêtre (miss. du Cons. de la Marine, 30 janv. 1717). Le cas du renvoi administratif fait l'objet d'une autre circulaire du Secr. d'Etat, 11 juin 1738. Lorsqu'il y a délit commun (cas privilégié) l'instruction est menée disait-on de conserve par le Supérieur de l'ordre et l'intendant, miss. 5 juill. 1782 cit. dans Moreau de Saint-Mery, Description topographique de la partie franscoise de l'isle de Saint-Domingue, t. VI, 262, 504.

${ }^{70}$ On dispose de nombreux cas «d'écarts de langage » de la part de religieux en matière de prônes et d'homélies lors des prédications : V. mise en garde du Secr. d'Etat, 19 avr. 1679, C.H.A.N. B9 $\mathrm{f}^{\circ} 34$.

${ }^{71}$ C.H.A.N. B12, ff ${ }^{\circ} 15$ ss (mémoire du roi aux Administrateurs Blénac- Du Maitz, 30 sept. 1686) demandant instamment confirmation d'une sentence reconnaissant la mise de ces religieux hors de cour par le Cons. souv..

${ }^{72}$ V. J. Janin, La religion aux colonies françaises..., Paris, 1942, 128 ; A. Gisler, ouvr. cit., 177 ( cad du P. Claude, capucin de Martinique ).

${ }^{73}$ Cf. arr. Cons. Sup. Cap 13 déc. 1762, qui condamne la morale et la doctrine des «soi disants (sic) Jésuites [parce que] on ne peut disconvenir que les negres soient leur trtoupeau chéri... », Arch. collège St Martial, Port-au-Prince, 10 - 18. ; contra : P. Lecrivain s.j. : «les jésuites ne prennent pas de position révolutionnaire... » Les missions jésuites, Paris, Découverte-Gallimard, 1991, 105. Mais le pouvaient-ils dans un Etat de droit, espace colonial soumis au $C$. N. à une époque où le gallicanisme montant et le jansénisme ne leur étaient pas favorables ?

${ }^{74}$ «On leur reproche dans cette colonie, et malheureusement ce reproche est un cri general et public, qu'ils s'occupent plus a tirer les nesgres a eulx qu'a la Religion; a les flatter qu'a les instruyre... il est notoire que les negres ont un atachement marqué pourles
} 
Marcel Chatillon a pu constater qu' "en aucun cas, il n'est question d'une instruction au rabais. Les jésuites, sur le plan religieux, se montrent profondément assimilationnistes, les esclaves participent aux mêmes offices, dans les mêmes églises avec le même rituel pour l'administration des sacrements. Un opuscule particulièrement didactique est imprimé par leurs soins pour la bonne compréhension des antiennes et hymnes liturgiques par les esclaves ${ }^{75}$. Il y a là selon le mot d'un auteur contemporain « un aspect subversif $^{76} »$.

Enfin, quant à l'action générale d'aide et d'assistance, la majorité des auteurs a soulevé la précarité et l'insuffisance évidente de la mission hospitalière de l'Eglise sous les tropiques. Précaire en France compte tenu des hordes de pauvres et de mendiants, l'œuvre de soin et de recueil des malades et des vieillards et agonisants aux Antilles n'est pas meilleure. Les religieux doivent se battre bec et ongles pour exiger que soit versées les oboles publiques ou privées servant à soutenir les hospices et autres mouroirs $^{77}$. La mentalité de l'époque, le peu de conscience des habitants et des agents royaux expliquent la multiplication des litiges entre laïcs et religieux. Cette conception très ancien régime de l'assistance publique perdura pendant une longue partie du $\mathrm{XIX}^{\mathrm{e}}$ siècle dans les colonies ${ }^{78}$ comme en métropole.

soi disants jesuites, ce qui ne peust estre que l'effet d'une conduicte indulgente. Ces religieux, de leur cotté, les payent en retour, et on ne peut disconvenir que les negres ne soient leur troupeau cheri... De tout tems ces religieux ont evité d'instruire les negres en presence de leurs mestres et des personnes libres; ils ont cherche a les separer des autres fidèles, a en former pour ainssy dire, un corps a part de fidèles, par l'etablissement d'une soi-disant cure des negres dans cette ville, et d'une espèce de fabrique (...). »

${ }^{75}$ Travail coll. de Chatillon, C. Fabre et J. Rosemain sur Une messe en cantiques pour esclaves in B. S. H. G., $\mathrm{n}^{\circ}$ 52, 2e trim. 1982. contra: R. Belenus, étude sur L'esclavage en Guadeloupe et en Martinique du XVII $a u_{-} X I X^{e} s$, Pointe-à-Pitre, éd. Jasor, 1998, 85 ss soulignant que la plupart des colons n'encouragent ni le mariage, ni l'évangélisation et que la mission du clergé se limite à prêcher la morale (...) ; malgré ce trop net raccourci l'auteur admet cependant «que seuls les Jésuites, dès 1684, donnent chaque dimanche une messe des nègres en leur église. »

${ }^{76} \mathrm{~V}$. Ph. Peyronnin, art. préc., 66.

${ }^{77}$ Cf. aff. J. Boury, sentence juge royal 14 sept. 1697 : le montant de la condamnation, rendue sur requête des religieux de l'hôpital S. Jean-Baptiste de la Martinique, soit 2000 lv de sucre, en application de l'art. 9 du C. N. mars 1685. J. Boury, mulâtre et libre prétend que cette disposition touchant les abus sexuels des maîtres sur leur esclave ne lui est pas applicable...

${ }^{78}$ Sur la quest.: V. G. Leti, Santé et société esclavagiste à la Martinique, 1802-1848, Ed. L'Harmattan, Paris, 1998. 
On l'aura compris : la loi de catholicité aux îles est intrinsèquement liée au phénomène d'exploitation et à l'évolution économique des Antilles. La prospérité des possessions françaises aux Amériques est fondée sur le travail obligatoire des colons engagés et l'esclavage des populations africaines : elle se devait d'avoir des limites et un garde fou. Louis XIII et Richelieu, les initiateurs, mais surtout Louis XIV, Colbert et les membres très advertis du Bureau des Colonyes en sont très conscients. L'aventure coloniale de la France reposait sur de solides fondements : dès 1679 une véritable administration royale s'applique sur les territoires lointains et les populations des Antilles mais on regrette que l'église antillaise coloniale ait été une église veufve ${ }^{79}$, comme l'on disait alors, c'est-à-dire, sans évêque, jusqu'en 1850. Cependant, la loi fondamentale de catholicité, introduite dès le début du XVII ${ }^{\mathrm{e}}$ siècle, s'étoffe et explique la multiplication des paroisses, des conseils de fabrique, l'installation d'un clergé étroitement surveillé par les préfets apostoliques et supérieurs vigilants, qui doit respecter l'ordre public royal en matière de religion dont le contrôle in terminis relève de l'intendant. Il semble honnêtement, à l'analyse, que l'on n'ait pas introduit tardivement les principes du catholicisme pour seulement moraliser la servitude, voire, en quelque sorte la légitimer ; en revanche, la législation royale, dès les origines, permettait d' envisager une colonisation en grande partie fondée sur le principe constitutionnel de catholicité (Ph. Sueur - P. C. Timbal) de la très grande majorité des sujets du royaume lequel à son tour légitimait, dans l'esprit du temps, l'action de la monarchie jusque dans les terres les plus lointaines d'Amérique.

\section{RESUME}

Le fait religieux aux Antilles et la règle fondamentale de la catholicité

Le fait religieux aux Antilles est avant tout pour l'historien et le juriste un fait social que l'on peut constater au quotidien. Il surprend encore le voyageur lorsqu'il pose pied et visite les anciens fleurons de la Couronne française. Or, l'histoire coloniale qui a forgé nolens volens l'âme de ces nouvelles contrées - et de ses

${ }^{79}$ Cf. Concile de Latran IV, 1215, can. 24 : « De electione facienda per scrutinum vel compromissum : ... et multa impedimenta proveniunt et magna pericula imminent ecclesiis viduatis... » 
habitants - repose tout naturellement sur la sujétion militaire et économique des Petites et des Grandes Antilles. Toutefois cette sujétion ne s'est pas toujours appuyée sur la force brutale ! Bien au contraire, elle entendit dès les premiers jours de la colonisation se fonder sur la mission religieuse voire spirituelle du nouvel occupant lequel agissait au nom du roi et des règles constitutionnelles de la monarchie. De plus, les quelques prêtres et surtout les religieux des principaux ordres, associés à l'oeuvre royale, se devaient non seulement de convertir les autochtones indiens puis les nègres déportés d'Afrique, mais de les éduquer, de les amender, de les instruire... en un mot d'en faire à long terme de bons sujets du roi. C'est sans doute la raison pour laquelle assez tôt certains ordres - et notamment les jésuites - revendiquèrent de s'occuper des esclaves. Malgré de très nombreux écrits, parfois contradictoires, il est difficile mais possible de dégager les fondements et les linéaments d'une politique religieuse en milieu colonial sous l'Ancien Régime.

Mots-clés : Antilles françaises, catholicité, esclavage, histoire coloniale, ordre colonial, religion

\begin{abstract}
The religious phenomenon in the French West Indies and the fundamental rule of Catholicity

The religious phenomenon in the French West Indies is, for the historian and for the jurist, primarily a social phenomenon observed on a daily basis. To this very day, it still surprises the traveller who sets foot on the centuries-old ornaments of the French Crown. But, the colonial history which forged (whether it was desired or not) the soul of these new lands - and their inhabitants - is quite naturally based on military and economic constraint exerted upon the Lesser and Greater Antilles. However, such constraint has not always been exerted under brutal force! Quite the contrary, as of the very first days of colonisation, it relied on a religious or spiritual mission of the new occupant, acting in the name of the King and the constitutional rules of the monarchy. Moreover, the few priests, and above all, the religious members of the major orders associated with the Royal task, were not only to convert the Indian natives and later the Negroes deported from Africa, but to raise them, change them, educate them ... in other words, over the long term, convert them into good subjects of the King. This is no doubt the reason why, very early on, certain religious orders - in particular, the Jesuits - claimed the soul-caring of the slaves. Despite numerous, at times contradictory, reports, it is difficult but possible to distinguish the basis and the guidelines of a religious policy in a colonial environment under the Ancien Regime.
\end{abstract}

Key Words: French West Indies, Catholicity, slavery, colonial history, colonial order, religion 